equal accumulated doses the incidence of leukæmia within ten months was anything between 6 and 30 per cent : dose-rate or radiation-free time certainly was a relevant factor. Ford and Mole $(P / 98)$ also showed elegant pictures of the chromosomal changes found in these leukæmic cells.

Malignant disease apart, a general non-specific shortening of life-span has been attributed to the effects of radiation. It could be postulated that radiation, either as a large single dose or a multiplicity of smaller doses, could act as a non-specific stress to give this result. Curtis and Gebhard $(P / 912)$ found differences in the patterns of mortality of irradiated and non-specifically stressed mice : in their hands the latter did not cause shortening of life. Following single large doses of radiation to the whole body, the earlier deaths are considered by Maisin et al. (P/109) to be the sequelæ of irradiation of the lung, the intermediate deaths to irradiation of the head and the latest to irradiation of the abdomen. Lindop and Rotblat $(P / 292)$ also reported their preliminary results in a very large experiment designed to elucidate some of the problems of ageing. Some of the observed effects were startling, which suggests that confirmation with standard strains of mice is highly desirable.

Malignant tumours and shortening of life result from the administration of radioactive materials giving substantial radiation-doses, the pathological changes being related to the chemical characteristics of the material given and its sites of concentration. The report of Burykina et al. $(P / 2077)$ presented by Krayevsky gives details of the late effects produced by administration of fission products to experimental animals. The title of their communication suggests that the doses were small, which is true in terms of mass, but the tissue-doses were measured in kilorads. Lamerton $(P / 61)$ reviewed the problems associated with the setting of permissible doses of radioactive materials. He believes that the estimation of the radiation-dose with all its present shortcomings provides the most satisfactory criterion. $\mathrm{He}$ argues that for the induction of osteo-sarcoma there is almost certainly a threshold which must be many hundreds of rads. Much of the argument is based on the one comprehensive experiment so far carried out on the long-term toxicological effects of radioactive materials; some aspects of this work in Chicago relating to strontium-90 were given by Finkel et al. $(P / 911)$.

Whereas radiobiologists are rightly most occupied at the present time with small doses of radiation and delayed effects, there is still considerable interest in the large single dose such as might be received in an accident, or which might have to be incurred by a few to avoid harm to many. Studies have been aimed at conditioning the body to resist radiation-doses normally lethal. This has not led to any practical measure which could safely be applied, but it has led to a better understanding of the effects of radiation on the intact mammal. Hollaender et al. $(P / 898)$ summarized their latest advances in this field of chemical protection. They favour the view that the main function of these agents is neutralization of free radicals formed by ionizing radiation. In the case of other active agents, biological amines, van der Meer et al. $(P / 556)$ argue convincingly in favour of tissue hypoxia. The role of oxygen in affecting the response of cells and tissues to ionizing radiations has been widely studied and Gray et al. (P/293) reviewed some of their recent work.

In addition to exploring the possibilities of protection against lethal doses, therapy of the animal lethally irradiated has been actively investigated. Hollaender et al. (P/898) reported their results of administering blood-forming cells which recolonize the marrow spaces of the irradiated animal. This grafting of homologous tissue may lead to undesirable late reactions. Barnes et al. $(P / 97)$, by cytological methods, have noted in some of these homologous chimæras a reversion of the marrow cells back to host type; apparently the cell clones with the greatest physiological competence, be they of normal donor or radiation-damaged host, ultimately predominate. In both laboratories these therapeutic exercises have involved administration of living cells to the irradiated mouse; but cell-free chemical agents are still being tried. Soska et al. $(P / 2121)$ claim reduced mortality from irradiation following administration of deoxy. nucleotides (but not following deoxynucleosides). Similarly, Panjevac et al. $(P / 490)$ found improved survival from the administration of deoxyribonucleic acid or ribonucleic acid from homologous spleen or liver, but not from testis.

Experimental work on the intact mammal has been chiefly the theme of this review. This does not mean that radiobiologists are now getting away from the simple systems - the root of the broad bean, the yeast cell and the bacterium. Many of the papers presented were concerned with the basic lesion in these test objects. Equally there were a number of papers primarily chemical, particularly on the chemistry of the nucleic acids; and a paper from the U.S.S.R. on effects of radiation on bio-lipids (Tarusov P/2248) indicates the continued interest in that country in the effects of radiation in the nervous system.

The collected papers will, of course, be published by the United Nations Organisation as the Proceed. ings of the Second Conference on the Peaceful Uses of Atomic Energy.
J. F. Loutit

\title{
PHYSICS OF QUANTA AND PARITY
}

\begin{abstract}
$\mathrm{T}$ HE opening session of Section A (Physics) of the British Association meeting in Glasgow on August 28 was devoted to papers presented by members of the Department of Natural Philosophy in the University of Glasgow. The research work of this Department is mainly concerned with studies of photomeson production and photodisintegration, utilizing the high-energy quanta which are provided by the departmental $340-\mathrm{MeV}$. synchrotron. Three
\end{abstract}

of the papers presented at this session were concerned with experimental investigations of this type; the fourth, by Prof. J. C. Gunn, professor of theoretical physics at Glasgow, discussed the important discovery during the past year of the non-conservation of parity.

In the opening address, Prof. P. I. Dee traced briefly the development of the concept of quanta from the original suggestion made by Planck, in 1901, 
to explain the conflict between the experimental results relating to the distribution of energy in black. body radiation and the straightforward predictions of classical theory in terms of the principle of equipartition of energy, which had been so fruitful in the development of the kinetic theory of gases. Compton's demonstration in 1923 that the wave-length changes which occur when X-rays are scattered by electrons could be quantitatively explained by treating the problem as a simple collision process, satisfying laws of conservation of energy and momentum, between a quantum of radiation and an electron, showed that the quantum concept applied to much wider fields than those in which absorption or emission of radiation were concerned.

The whole of the physical world, as we know it, may be regarded as comprised of matter and radiation. It is natural, therefore, that the interactions between the elementary quanta of radiation and the basic constituent particles of matter, neutrons and protons, should constitute one of the frontier problems in modern physics. In processes such as these, mesons are created, and in view of the part played by these particles in relation to nuclear forces, experiments of this nature are therefore not only fundamental to the understanding of the basic phenomena of interaction between radiation and matter but also shed light upon the structure of atomic nuclei.

Recent experiments at Glasgow and elsewhere upon the photoproduction of $\pi^{+}$and $\pi^{-}$mesons in hydrogen and deuterium permit the comparison of the crosssections for the production of these particles in reactions of quanta with protons and neutrons, respectively. In these experiments the important principle that nuclear forces are independent of charge seems to receive good confirmation. Similar experiments upon the relative production of $\pi^{+}$ mesons in hydrogen and deuterium provide information upon the dependence on spin of the photomeson production interaction. Studies of $\pi^{0}$ production by quanta in helium suggest that in this process the close proximity of other nucleons has little effect upon the primary interactions between the quanta and the elementary nucleons.

The idea of quantization in physics applies, of course, in a much more profound and general manner than merely in relation to the electromagnetic radiation. The concept of structure in the intervening medium was introduced long ago by Faraday to explain the forces between electrical charges. This concept of the 'field' in physics has now assumed a very wide significance. It is even possible that all the elementary particles of matter, the protons, neutrons, mesons, etc., consist essentially of particular quantized states of a general field. Of what this field consists no one at present can say ; indeed, it may not yet even be sensible to inquire.

In the following paper, Mr. J. M. Reid (Glasgow) gave an account of recent work upon the disintegration of nuclei under irradiation by high-energy quanta. Experiments of this nature have been made with quanta over a wide range of energies, from a few $\mathrm{MeV}$. up to about $300 \mathrm{MeV}$. At the lower end of this energy region the main feature in the case of all nuclei is a broad or 'giant' absorption resonance which reaches its maximum at a quantum energy of about $20 \mathrm{MeV}$. and has a width of about $6 \mathrm{MeV}$. This resonance has been explained as a collective oscillation in which all the protons in the nucleus move together in one direction while all the neutrons move in the other. Such a picture can only provide a. rough indication of nuclear behaviour, since it omits all details of the motion of the proton and neutron 'fluids'. Indications have been found of departures from the bell-shaped resonance curve which such a model predicts. In particular, a cloudchamber investigation at Glasgow has shown strong absorption of quanta of very sharply defined wave-length at the low-energy end of the 'giant' resonance.

A similar cloud-chamber experiment has confirmed a result obtained in American laboratories by other techniques. This concerns the energy region above the 'giant' resonance where, by virtue of its short wave-length, the quantum energy is so localized that only part of the nucleus is affected. A sub-unit greatly favoured in such interactions has been shown to be a single proton-neutron pair. These two nucleons are, as a result, violently ejected as a free neutron and proton, apparently uninfluenced (at least in a large number of cases) by the presence of the rest of the nucleus. Similarly, when this happens, the rest of the nucleus continues in its state of motion despite the plucking of the pair of particles from within its volume. This seems to open up the possibility of investigating, in a more direct way than hitherto, the nature of the internal motion which is occurring within the atomic nucleus.

Dr. J. G. Rutherglen (Glasgow) delivered a lecture devoted to the techniques and uses of millimicrosecond measurements, which have now assumed great importance in atomic and nuclear investigations. For example, the new fundamental particles which have been discovered in the past ten years are all unstable and only live for times between a microsecond and a ten-thousandth of a microsecond. There are many important processes in nuclear physics which take place in comparable, or even shorter, times than this. Measurements of very short timeintervals are therefore necessary to investigate the properties of these particles and processes which are fundamental to our understanding of the basic properties of matter.

The electronic techniques involved in measurements in the region down to a microsecond were developed to a very high degree during the Second World War by the needs of radar and similar applications. At that time the measurements in the field of nuclear physics were limited to about a microsecond by the properties of Geiger counters and similar devices which were then available for the detection of nuclear radiations.

During the past ten years both the detectors and the electronic techniques have been improved in regard to time resolution by a factor of the order of one thousand, so that it is now possible to measure time-intervals as short as one millimicrosecond and to detect even shorter differences in time. The main advance in the detection technique has been the development of the scintillation counter, in which part of the energy of the nuclear radiation is converted into light in a block of transparent material, and the light flash is converted into an electrical pulse by means of a photomultiplier. Techniques of this nature are now commonly employed in many nuclear investigations. For example, $\pi+$ mesons are often identified by measuring the time-interval, of the order of $1 / 50$ microsecond, between the pulse due to the arrival of the $\pi+$ meson and that due to the $\mu+$ meson which results from its decay. Again, the life-times of short-lived nuclear states may in some 
cases be measured in similar fashion, but the order of time resolution which is so far possible is not yet sufficient for very comprehensive investigations in this particular field.

The second main topic at the opening session was "The Non-Conservation of Parity", which was very clearly presented both in respect of its difficult primary concepts and of its fundamental influence upon basic physical principles by Prof. J. C. Gunn. The recent discovery, following a suggestion of Lee and Yang, that parity is not conserved in certain weak elementary particle interactions, represents a revolution in our ideas of space and time in some ways comparable with the introduction by Einstoin of his special theory of relativity in 1906. The essence of Einstein's idea was that the laws of all natural phenomena should be the same for all observers in uniform motion relative to each other. It was automatically assumed that along with this invariance of the equations of physics for transitions to moving reference frames would go invariance to reflexions in space and time. In particular, invariance to reflexions in space is mathematically expressed as the conservation of parity. Put in its simplest form, such invariance simply means that the mirror-image of any physical or chemical system shows a behaviour which could occur in accordance with natural laws in the real world. Effectively, the scientist looking at any experiment cannot say whether what he is seeing is the real thing, or a reflexion in a mirror.

It is indeed true that parity conservation, or invariance to reflexion, is observed for all the more ordinary physical processes-the processes controlled by electrical forces, which account for the physics and chemistry of everyday life, and the processes of more recent interest controlled by nuclear inter- actions. Of course, local conditions, as, for example, biological systems on Earth, may result in only, say, the right-handed form of some molecule or structure occurring, its left-handed mirror-image being absent. However, this is only a local phenomenon and the left-handed variety is, in principle, quite possible.

This is how things rested when Lee and Yang made their proposal that, though invariance to reflexion. holds for the strong electromagnetic and nuclear interactions, it might not hold for weak interactions, such as those responsible for $\beta$-decay of nuclei and other decay processes of the unstable particles found in high-energy physics. Their hypothesis has been brilliantly confirmed by experiments, both on $\beta$-decay and on the decay of unstable particles, and it is now established that parity conservation is violated over the whole range of weak decays. For example, the neutrino emitted in $\beta$-decay always spins like a lefthanded screw along its direction of motion. The mirror-image of this would be a right-handed screw neutrino and this is not found to exist.

This left-handedness of the neutrino provides a means by which the physicist can now discriminate between a real and a looking-glass world. An important reservation must, however, bo made-the mirrored image of a decay experiment with ordinary matter is impossible as a real process for ordinary matter, but, according to current belief, it represents exactly how anti-matter would behave. Thus, if, possibly in other galaxies, there are worlds of antimatter their neutrinos are right-handed in our sense. In view of the possible existence of such worlds, no universal significance can be expressed to lefthandedness. We might indeed say that, like that of biological systems, the handedness of the neutrino is an environmental character, but the environment is a wide one, consisting of all ordinary matter.

\section{MICROWAVE PHYSICS}

$\mathrm{T}$ HE final session of Section $A$ of the British Association meeting in Glasgow was held on September 3, and was devoted to the subject of microwave physics. It was appropriate that the chair should be taken by Dr. B. J. Mason, for he has acted as recorder of this Section for a number of years and now retires from this onerous and responsible position. The meeting warmly expressed their appreciation of his work.

There were three speakers, and it was soon clear that their papers had been planned to try to give, for the non-expert, some indications of the remarkable developments which have occurred in microwave physics since the War. The first speaker, Prof. K. W. H. Stevens, of the University of Nottingham, began his lecture by giving an elementary account of what is meant by electron spin resonance. The magnetic moment associated with the spin of the electron has two equilibrium positions in a magnetic field, of different energies. The moment can be turned from the lower one to the higher one by supplying a quantum of electromagnetic radiation of the right magnitude. For a free electron the relation between the size of the field and the wave-length of the radiation is $H \lambda=10 \cdot 70$, where $H$ is measured in kilo-oersteds and $\lambda$ in $\mathrm{cm}$. For fields of order $3 \times 10^{3}$ oersteds, a convenient laboratory magnitude, $\lambda$ is approximately $3 \mathrm{~cm}$., which is in the microwave region. Very good oscillators and components are available for these wave-lengths, as a result of wartime radar developments.

Although all substances contain many electrons these are usually paired off, with spins opposed. Such pairs do not show electron spin resonance. Thus to demonstrate the resonance it is essential to work with substances containing unpaired electrons, and it seemed, at one time, that this would provide a serious limitation to the use of the spin resonance technique. This has not in fact proved to be the case, for it is now clear that unpaired electrons occur very widely, and one of the great virtues of the technique is that it can investigate these electrons in detail and thus throw light on problems which are of considerable interest and which are not always easily investigated by other means.

This aspect was particularly emphasized in the second part of Prof. Stevens's talk and by Dr. D. J. E. Ingram, of the University of Southampton, who followed him. The main idea is to use the electron magnetic moment as a probe to find out something about the electron motion. In the course of its motion, the electron may move from one atom to 